Meta

Journal des traducteurs

Translators' Journal

\title{
Stratégies de traduction : les introductions et les conclusions dans des textes de vulgarisation scientifique
}

\section{Joëlle Rey et Mercedes Tricás}

Volume 51, numéro 1, mars 2006

URI : https://id.erudit.org/iderudit/012871ar

DOI : https://doi.org/10.7202/012871ar

Aller au sommaire du numéro

Éditeur(s)

Les Presses de l'Université de Montréal

ISSN

0026-0452 (imprimé)

1492-1421 (numérique)

Découvrir la revue

Citer cet article

Rey, J. \& Tricás, M. (2006). Stratégies de traduction : les introductions et les conclusions dans des textes de vulgarisation scientifique. Meta, 51(1), 1-19. https://doi.org/10.7202/012871ar
Résumé de l'article

Cette étude a pour objectif d'examiner les stratégies interprétatives mises en oeuvre pour traduire les introductions et les conclusions de textes de semi-vulgarisation scientifique. En effet, l'analyse d'éléments textuels déterminés, comme les signes qui contribuent à la construction d'un réseau de cohérence, les mécanismes d'agencement des segments et les marques de polyphonie et notamment le degré de spécialisation du lexique employé révèlent que, dans ces deux blocs textuels, le langage a une fonction essentiellement argumentative qui contraste avec la fonction informative de la partie centrale du texte.

La non prise en compte de ces spécificités lors du processus de traduction peut introduire des modifications au niveau de la fonction pragmatique, du point de vue et de l'intention de l'auteur du texte original. L'interprétation du texte devrait donc intégrer les sens primaires et secondaires, les valeurs et les fonctions de ces deux espaces textuels pour que le texte traduit reproduise la même fonction d'introduction et de conclusion que l'original.
Ce document est protégé par la loi sur le droit d'auteur. L'utilisation des services d’Érudit (y compris la reproduction) est assujettie à sa politique d'utilisation que vous pouvez consulter en ligne.

https://apropos.erudit.org/fr/usagers/politique-dutilisation/ 


\title{
Stratégies de traduction: les introductions et les conclusions dans des textes de vulgarisation scientifique
}

\author{
JOËLLE REY \\ Universitat Pompeu Fabra, Barcelone, Espagne \\ joelle.rey@upf.edu \\ MERCEDES TRICÁS \\ Universitat Pompeu Fabra, Barcelone, Espagne \\ merce.tricas@upf.edu
}

\begin{abstract}
RÉSUMÉ
Cette étude a pour objectif d'examiner les stratégies interprétatives mises en œuvre pour traduire les introductions et les conclusions de textes de semi-vulgarisation scientifique. En effet, l'analyse d'éléments textuels déterminés, comme les signes qui contribuent à la construction d'un réseau de cohérence, les mécanismes d'agencement des segments et les marques de polyphonie et notamment le degré de spécialisation du lexique employé révèlent que, dans ces deux blocs textuels, le langage a une fonction essentiellement argumentative qui contraste avec la fonction informative de la partie centrale du texte.

La non prise en compte de ces spécificités lors du processus de traduction peut introduire des modifications au niveau de la fonction pragmatique, du point de vue et de l'intention de l'auteur du texte original. L'interprétation du texte devrait donc intégrer les sens primaires et secondaires, les valeurs et les fonctions de ces deux espaces textuels pour que le texte traduit reproduise la même fonction d'introduction et de conclusion que l'original.
\end{abstract}

\begin{abstract}
The aim of this study is the examination of interpretation strategies developed by the translator when dealing with introductions and conclusions in semi-specialized scientific texts.

The analysis of specific textual items, such as coherence devices, connective mechanisms and polyphonic marks and also the degree of specialization of the words used in these two specific textual spaces show that language has mainly an argumentative function as opposed to the informative function of the central part of the text.

If the translator does not take into account these considerations, the pragmatic function, the point of view and the intention of the author can be altered. Therefore, the interpretation of a text should carefully process the primary and secondary meaning, the values and functions of these parts of the text in order to convey the introductory and conclusive function of the original.
\end{abstract}

\section{MOTS-CLÉS/KEYWORDS}

analyse textuelle, textes de spécialité, cohérence, fonction, intentionnalité

L'hypothèse de départ que nous formulons dans cet article est que les introductions et les conclusions sont des blocs textuels spécifiques, dont les caractéristiques et la fonction doivent être identifiées et prises en compte dans toute opération d'interprétation du texte, que ce soit dans le cadre de l'analyse textuelle ou de la traduction. 
En effet, la lecture de textes originaux et de leur traduction révèle que les traducteurs, focalisant leur attention sur le transfert des éléments informatifs, ont trop souvent tendance à appliquer des stratégies interprétatives globales et non différenciées à l'ensemble du texte, sans se rendre compte que les introductions et les conclusions exigent un traitement spécifique car elles sont situées à deux endroits clés: l'ouverture et la clôture de l'acte de communication. L'importance de cette position est confirmée par les études menées sur les stratégies de lecture dans différents types de textes (Jacobi 1987; Adam 1997) qui montrent que l'attention portée par le lecteur à l'introduction et la conclusion font généralement l'objet d'une lecture particulièrement attentive.

Pour mieux cerner les spécificités des introductions et des conclusions, nous avons constitué un corpus de textes scientifiques qui nous a permis de définir quels types de stratégies entrent en jeu dans ces blocs textuels et de montrer que la construction du sens ne se limite pas à la partie informative mais englobe une série de phénomènes interprétatifs que le traducteur ne doit pas négliger.

Les textes analysés sont pour la plupart des articles parus dans la revue française La Recherche entre 1998 et 1999, dont la traduction espagnole a été publiée dans Mundo Científico. Nous avons sélectionné une trentaine d'articles du même type - des «articles courts», de deux à trois pages -, mais traitant de sujets divers pour que la validité de l'analyse ne semble pas limitée à un domaine de spécialité déterminé. Dans le même esprit, et pour montrer que les mêmes phénomènes pouvaient être observés dans d'autres types de publications, nous avons intégré au corpus cinq textes de semivulgarisation scientifique publiés en 1997 dans plusieurs numéros du Courrier de l'Unesco.

Une comparaison minutieuse du texte original et de la traduction a permis d'identifier les segments à analyser d'après les critères de sélection suivants: a) les éléments du texte traduit qui, ne reproduisant pas une traduction littérale, montrent une création du traducteur, résultat d'un acte interprétatif élaboré, et b) la nature argumentative de ces éléments.

La Recherche et Mundo Científico sont des revues qui jouissent d'un certain prestige et s'adressent non seulement à des chercheurs mais aussi à des lecteurs ayant des connaissances scientifiques solides et désireux de suivre l'évolution des recherches dans les différents domaines. Il s'agit donc de publications de semi-vulgarisation scientifique qui ne sont pas distribuées en circuit fermé, comme peuvent l'être des revues réservées à des spécialistes, mais qui sont vendues en kiosque ou par abonnement et, par conséquent, s'adressent à un public beaucoup plus large. Dans un tel contexte, les stratégies visant à attirer l'attention du lecteur et à l'inciter à lire acquièrent une importance particulière, et un texte destiné à transmettre des connaissances devient un espace d'interaction dans lequel les mécanismes rhétoriques jouent un rôle important.

L'organisation rhétorique de ces articles, la façon de combiner les espaces informatifs et les espaces intentionnnels, la manière d'indiquer l'ouverture et la clôture, contribuent à mettre en évidence la nature essentiellement argumentative des introductions et des conclusions, qui contraste avec la prédominance de l'informativité dans la partie centrale du texte.

Cet ensemble de traits caractéristiques - une organisation particulière, le fait de combiner informativité et intentionnalité, l'utilisation spécifique de certains éléments 
linguistiques - nous conduit à affirmer qu'introduire et conclure sont deux actions textuelles autonomes, qui se différencient du reste du texte.

On pourrait même considérer qu'elles constituent un acte de parole particulier, caractérisé par une intentionnalité spécifique et une cohérence autonome. Une telle différence est due essentiellement à la nature du langage utilisé dans ces parties initiale et finale par comparaison au reste du texte.

L'analyse du corpus confirme cette divergence qui nous paraît importante et que nous résumerons en ces termes:

Dans les introductions et les conclusions des textes de vulgarisation scientifique le langage a une fonction essentiellement argumentative alors que dans la partie centrale il a une fonction essentiellement informative.

Il est généralement admis que les textes de vulgarisation scientifique transmettent des notions objectives, des concepts techniques et des prémisses vérifiables. Or, dans les introductions et les conclusions, on observe fréquemment que les chercheurs s'efforcent de se rapprocher du lecteur pour éveiller son intérêt ou faire appel à ses émotions. En effet, dans ces deux blocs textuels, on relève une fréquence plus élevée de mouvements de type évaluatif, introductif, récapitulatif, visant à persuader le lecteur, à le convaincre et à insister sur l'intérêt et l'exactitude de ce qui a été exposé. C'est pourquoi la traduction de ces espaces exige des stratégies interprétatives adaptées à leur spécificité.

Dans les traductions des textes analysés, on observe à plusieurs reprises que le traducteur ne distingue pas ces deux fonctions du langage, ce qui donne comme résultat des interprétations erronées qui modifient les intentions de l'auteur: alors que ce dernier employait un registre courant pour attirer l'attention du lecteur et le convaincre de la pertinence de ses affirmations, le traducteur a opté pour l'utilisation de mots qui, bien que sémantiquement proches de ceux du texte de départ, n'ont pas la force émotive de l'original et, au contraire, sont associés au monde objectif et rigide de la science dont l'auteur voulait s'éloigner dans ces segments.

Pour analyser cette spécificité des espaces textuels introductifs et conclusifs, nous appliquerons des formulations et des concepts empruntés à la théorie de l'argumentation dans la langue, développée par le groupe de linguistes dirigé par O. Ducrot. Nous considérons que certaines notions dégagées par ces théoriciens, et en particulier la différence qu'ils établissent entre argumentativité et informativité - étroitement liée à l'opposition entre langage naturel et langage logique -, peut être utile pour expliquer les raisons pour lesquelles le traducteur n'applique pas toujours la stratégie interprétative adéquate à ces deux blocs textuels.

Dans ses lignes générales, la théorie de l'argumentation affirme que tout acte discursif est essentiellement intentionnel et reflète les sentiments et les pensées de ses auteurs. Les textes sont interprétables parce que les relations qui les articulent répondent à des schémas intentionnels. Sans la présence d'un principe argumentatif qui relie et structure l'ensemble, tout acte de compréhension et d'interprétation serait impossible.

L'argumentation apparaît donc comme une notion purement linguistique - et non pas uniquement discursive - fondée sur la nature même du langage et sur son caractère relationnel. Défendre la capacité de la langue à organiser et à structurer des points de vue revient à reconnaître que les mots sont porteurs d'attitudes, d'intentions, de 
sentiments et de positionnements. L'intentionnalité que le sujet parlant met en œuvre devient une activité linguistique qui s'appuie sur une base nettement sémantique, car elle surgit d'instructions insérées dans les signes linguistiques. Sémantique et pragmatique deviennent alors deux niveaux d'analyse qui, loin d'être opposés, sont complémentaires et interdépendants.

Les conceptions argumentativistes ont réussi à dissocier le concept de sens de la notion d'information à laquelle il avait longtemps été lié. À partir d'une critique lucide de concepts comme la référence et la dénotation, qui correspondent à ce que Ducrot appelle le paradigme informatif, la théorie de l'argumentation remet en question les bases de la sémantique traditionnelle - et sa vision des mots considérées comme des compartiments étanches qui découpent des portions de réalité - pour affirmer que les mots de la langue naturelle, tels qu'on les utilise couramment, ne reflètent pas des concepts mais des points de vue.

Le sens, dissocié ainsi de la notion d'information, acquiert un caractère essentiellement intentionnel, dans la mesure où le locuteur peut choisir, délimiter, orienter ce qu'il veut dire. Selon O. Ducrot, toute activité énonciative est animée par l'intention de modeler les objets du discours, ce qui l'amène à affirmer :

Je n'arrive pas à concevoir un sens qui ne soit pas la mise en œuvre d'une intention argumentative. (Ducrot 1982: 158)

Les thèses d'O. Ducrot et de ses disciples ont démontré la prépondérance de l'aspect argumentatif de la communication au détriment de la composante informative, au point d'aboutir à l'argumentativisme proclamé par la théorie des topoï.

Cette notion de topoï, étroitement liée à celle de point de vue, permet un traitement sémantique des phénomènes pragmatiques et constitue un instrument interprétatif puissant que le traducteur peut utiliser comme guide dans son travail.

Grâce à la présence des schémas topiques, les éléments lexicaux, loin de constituer un simple miroir de la réalité, véhiculent des points de vue car

[...] ils reflètent à la fois les croyances et les intentions des locuteurs. (Raccah 1990: 194)

L'utilisation du langage à des fins scientifiques, comme c'est le cas dans notre corpus, pourrait contredire une telle approche. En effet, les discours scientifiques tendent à une plus grande objectivité et l'expression de points de vue, de croyances et de jugements de valeur ne semble pas y être prédominante. On pourrait même penser que le langage utilisé exige une objectivité absolue.

Raccah (2000b) tente de résoudre la contradiction entre subjectif et objectif dans le lexique en établissant une nette distinction entre deux types d'éléments lexicaux: les mots et les termes. Il désigne par «termes» les éléments linguistiques qui reflètent l'objectivité propre au langage spécialisé, et par «mots» les éléments que le sujet parlant emploie pour communiquer et dont il assume la responsabilité de façon individuelle. Bien que, d'un point de vue formel, les «termes» fonctionnent comme des mots et se comportent syntaxiquement comme tels, d'un point de vue sémantique ils ne correspondent pas vraiment à la catégorie de «mots» car ils sont dépourvus d'orientation argumentative.

Pour illustrer cette différence entre «mots» et "termes», Raccah souligne le caractère insolite d'une situation communicative dans laquelle un locuteur, utilisant la forme habituelle du langage, dirait: 
Il fait froid dans cette salle.

et recevrait une réponse formée par des «termes» au lieu de «mots»:

La température est de sept degrés.

La réponse est bizarre car généralement, dans la communication quotidienne, le mot «froid» ne prétend pas exprimer la mesure objective d'une température mais une perception subjective de cette réalité. La réponse donnée dans cet exemple montre que l'interlocuteur a confondu l'utilisation de «froid» comme «mot» et comme «terme».

Interpréter un "mot» comme un «terme» et privilégier sa valeur informative au détriment de sa valeur argumentative peut avoir, comme nous le verrons, des conséquences importantes en traduction.

En effet, lorsqu'un traducteur, ne percevant pas que l'exigence de rigueur scientifique est parfois remplacée par un type de communication plus proche et plus interpersonnel, traduit des mots par des éléments moins évocateurs - même s'ils semblent plus appropriés dans ce genre de discours -, il applique une stratégie de traduction erronée.

Dans les introductions et les conclusions des articles de vulgarisation que nous avons analysés, les scientifiques s'efforcent d'utiliser des instruments linguistiques dont les connotations émotives et persuasives permettent d'impliquer le lecteur d'une façon plus directe. Or, à plusieurs reprises, les traducteurs ont mal interprété les schémas topiques des mots et, par là même, le point de vue de l'auteur. Alors que ce dernier prétendait utiliser le langage naturel pour attirer l'attention du lecteur et le convaincre du bien-fondé de ses affirmations, le traducteur a opté pour l'utilisation de mots qui, bien que sémantiquement proches de l'original, n'ont pas le même potentiel argumentatif.

Cette modification du point de vue de la part du traducteur est parfaitement illustrée par l'exemple suivant extrait du corpus: la traduction du mot français «énigme» par problema dans le texte espagnol.

Les sismologues s'attèlent à résoudre l'énigme ${ }^{1}$.

Los sismólogos trabajan intensamente en la resolución del problema.

Il semble évident que "énigme» et problema transmettent des points de vue et des nuances intentionnelles différents et traduire l'un par l'autre n'est pas exempt de conséquences. On peut aisément montrer que, du point de vue sémantique, ces deux éléments lexicaux ne sont pas coïncidents et interchangeables car leurs schémas topiques diffèrent sensiblement. Dans le texte français, la capacité argumentative d'un mot comme «énigme» permet de déployer des instructions de jeu, de complicité, de défi. En le remplaçant par problema, le traducteur a opté pour une tendance à l'objectivité plus prononcée sans prendre en compte que, dans l'espace conclusif où ce mot apparaît, l'auteur prétendait justement atténuer la rigidité qui caractérise ce type de textes.

Dans leur perception du caractère relationnel du sens, O. Ducrot et ses disciples accordent aussi un rôle fondamental à des éléments d'agencement dont la fonction consiste à établir des relations de cohérence inter et intra discursives: les connecteurs et les opérateurs argumentatifs. Leur fonction primordiale consiste à établir des restrictions et des régulations nettement sémantiques à travers lesquelles ils contribuent 
de façon substantielle à l'interprétation pragmatique des productions discursives. Leur capacité d'établir des liens sémantico-pragmatiques est telle qu'ils deviennent des piliers fondamentaux de l'acte interprétatif car

Ils ajoutent à leur fonction de liaison une fonction de mise en relation argumentative. (Amossy 2000: 159)

Les connecteurs discursifs ont pour mission de guider les inférences réalisées dans la communication et, par conséquent, contribuent de façon essentielle à la compréhension d'un texte.

Un autre outil conceptuel développé par O. Ducrot (1980, 1984) peut ouvrir au traducteur des voies d'interprétation particulièrement utiles: il s'agit du concept de «polyphonie».

La théorie de la polyphonie présente le locuteur comme le responsable ultime de l'acte communicatif:

j'entends par locuteur un être qui, dans le sens même de l'énoncé, est présenté comme son responsable, c'est-à-dire comme quelqu'un à qui l'on doit imputer la responsabilité de cet énoncé. (Ducrot 1984: 193)

Le locuteur organise et distribue les rôles des différentes voix qui prennent la parole pour exprimer des points de vue différents. La polyphonie devient ainsi la propriété discursive qui permet au locuteur de présenter un texte comme un ensemble hétérogène. L'analyse des mécanismes polyphoniques révèle, sous l'apparence monolithique de l'acte textuel, un espace de dialogue et de négociation dans lequel interviennent des énoncés divers et asymétriques, des mouvements argumentatifs distincts dont la conjonction conforme la complexité énonciative. De ce point de vue, les introductions et les conclusions constituent deux espaces polyphoniques clairement différenciés de la partie centrale du texte.

Les traits de ces blocs textuels - une fréquence plus élevée de jugements de valeurs, l'intention de convaincre le lecteur et d'établir un contact plus direct avec lui... - peuvent s'expliquer par l'entrée en scène d'un énonciateur qui, abandonnant le rôle de scientifique rigoureux, devient un personnage animé par une volonté didactique et un désir de se rapprocher du public, ce qui le pousse à utiliser des instruments linguistiques appartenant à un autre registre.

Et, évidemment, si l'utilisation du langage par ces énonciateurs est perçue comme étant différente, le traitement interprétatif appliqué par le traducteur doit s'adapter à cette différence.

Mais ce jeu polyphonique des introductions et des conclusions ne se limite pas à l'intervention de deux énonciateurs; ces deux blocs textuels se distinguent, en outre, du reste du texte par leur capacité de déployer une multiplicité de voix secondaires. Chacune de ces voix ouvre son propre cadre argumentatif et délimite de nouveaux points de vue: les citations, les questions, les discours directs et tous ces phénomènes que Authier-Revuz (1982) regroupe sous la dénomination de polyphonie «constitutive» sont des marques discursives qui signalent la présence de ces énonciateurs secondaires et exigent des instruments interprétatifs adéquats.

Si le traducteur n'est pas capable de découvrir ces stratagèmes rhétoriques, il ne percevra pas la présence de virages prononcés sur un trajet qu'il imagine beaucoup plus linéaire et tombera dans le piège d'interpréter certains segments d'une façon erronée. 
Avant de traduire, il est donc nécessaire d'identifier les principaux mécanismes sur lesquels s'appuie la construction des introductions et des conclusions que nous avons résumés comme suit:

a) la structure argumentative qui donne forme à l'ensemble textuel et à chacune de ses parties;

b) les réseaux de cohérence qui transforment une série d'éléments verbaux en un acte de communication;

c) la structure polyphonique qui présente le texte comme le lieu de rencontre d'une série de voix ayant des caractéristiques spécifiques et des exigences interprétatives différenciées;

d) la combinaison d'éléments rhétoriques qui donnent forme à la matière verbale et constituent le point de départ du processus interprétatif.

La présence élevée de ces mécanismes dans ces deux blocs textuels indique aussi que les introductions et les conclusions, bien qu'ayant une fonction textuelle opposée, conservent une certaine symétrie, tant au niveau thématique qu'au niveau interactionnel, une caractéristique qu'il conviendra de prendre en compte lors de la traduction.

\section{Analyse des introductions}

Dans les textes que nous avons analysés, l'introduction a essentiellement pour fonction:

- de compléter le rôle d'accroche du titre, du sous-titre, et du chapeau, en utilisant une palette de mécanismes rhétoriques destinés à capter l'attention du lecteur;

- d'introduire le thème de l'article, c'est-à-dire d'entrer en matière de façon graduelle, sans sursaut, afin d'éviter un rejet éventuel de la part du lecteur qui trouverait le sujet beaucoup trop ardu.

De cette dernière caractéristique découle un problème de délimitation des introductions. En effet il n'y a pas à proprement parler de marqueur qui puisse indiquer la fin d'une introduction et il est souvent difficile de savoir exactement où se termine l'introduction et où commence le corps du texte. Selon les normes standard de rédaction, l'introduction devrait correspondre, en principe, au premier paragraphe du texte; la fin de l'introduction serait donc marquée typographiquement par un point et un alinéa. Or, dans les textes étudiés, on observe que, dans de nombreux cas, la présentation du thème se prolonge sur deux ou même trois paragraphes, s'intégrant peu à peu au texte. En fait, on perçoit qu'on est passé de l'introduction au corps du texte à mesure que s'estompent des éléments caractéristiques de ce bloc textuel que nous avons classés en trois grands groupes:

1. Les signes qui contribuent à la construction d'un réseau de cohérence spécifique, parmi lesquels nous distinguons: les éléments lexicaux, les mécanismes cataphoriques et les jeux métaphoriques qui établissent un lien entre le langage spécialisé et le langage général.

2. Les mécanismes d'agencement des segments, particulièrement ceux qui produisent un effet d'immédiateté et d'interactivité.

3. Les marques de polyphonie, qui permettent l'intervention de voix appartenant à des domaines autres que scientifique et à travers lesquels l'auteur transmet des points de vue plus personnels et subjectifs. 


\subsection{Le lexique comme ensemble de signes qui contribuent à la construction d'un réseau de cohérence spécifique}

La lecture d'un article scientifique ou de tout autre texte considéré comme informatif doit permettre au lecteur d'intégrer des connaissances nouvelles dans des connaissances déjà acquises. Ce processus cognitif assez complexe ne peut se réaliser de façon satisfaisante que si les connaissances nouvelles sont présentées de façon progressive et constamment mises en rapport avec des éléments connus. C'est pourquoi, dans les introductions, les concepts présentés sont plutôt généraux et la densité de terminologie spécialisée est plus faible que dans le reste du texte. En fait, les termes de spécialité sont introduits graduellement par le biais de mécanismes divers comme, par exemple, les mécanismes cataphoriques qui permettent d'employer d'abord un mot de la langue courante le plus descriptif possible qui sera associé par relation cataphorique à un terme plus spécialisé.

Ainsi, dans le texte Le noyau de la terre tourne-t-il vraiment $?^{2}$, ce noyau interne, appelé «la graine», est d'abord présenté comme une «sphère solide située au centre de la terre». Les comparaisons, les métaphores et, de façon générale, les mécanismes sur lesquels reposent les opérations d'analogie sont fréquents dans l'introduction.

Or, l'analyse du corpus révèle que cette volonté de l'auteur d'éviter un registre spécialisé afin d'établir une certaine complicité avec le lecteur, n'est pas toujours perçue ou prise en compte par le traducteur. En effet, ce dernier, considérant qu'un texte spécialisé exige un registre spécialisé, a tendance à remplacer des «mots» par des «termes».

Cette tendance apparaît très nettement lorsque le traducteur doit expliciter certains éléments. Dans l'exemple ci-dessous, la phrase en français est séparée en deux segments: un premier segment mentionne une «idée» et un second segment, séparé du premier par deux points, explique en quoi consiste cette "idée». Cette forme synthétique, dans laquelle le rapport entre les deux segments reste implicite, ne se prête guère à la traduction littérale en espagnol. Aussi, le traducteur a-t-il réorganisé les éléments pour que le texte espagnol semble plus naturel, ce qui l'a conduit à proposer:

idée [...]: les charges

idea que [...]. Según esta hipótesis, las cargas...

Comme la plupart de ses collègues, il avait même écarté cette idée qui, depuis quelques années, agitait les marges de ce domaine de recherche: les charges se déplaceraient librement au sein de ces matériaux dans un paysage organisé en bandes larges de quelques atomes ${ }^{3}$.

Como la mayoría de sus colegas, había descartado incluso una idea que años antes se movía por los márgenes de este campo de investigación. Según esta hipótesis, las cargas se moverían libremente por el interior de un paisaje formado por bandas de varios átomos de anchura.

Le fait de séparer les segments et d'expliciter le lien entre les deux a obligé le traducteur à opérer, dans le second segment, une reprise anaphorique. Comme résultat, ce qui apparaissait dans le premier segment comme «une idée» qui agitait les marges de ce domaine de recherche devient une «hypothèse», terme très utilisé dans 
le langage scientifique. Or le mot «hypothèse» implique quelque chose de beaucoup plus solide, beaucoup plus élaboré qu'une simple «idée», alors que précisément l'auteur utilise le mot «idée» pour souligner qu'il s'agit de quelque chose qui, en principe, semblait anodin. Dans ce cas, s'il est évidemment préférable d'expliciter le lien entre les deux énoncés en introduisant l'expression "según", il n’était pas nécessaire de reprendre le mot «idée» et on pouvait tout simplement remplacer les deux points par une virgule et enchaîner en utilisant l'expression «y según la cual».

Como la mayoría de sus colegas, había descartado incluso una idea que años antes se movía por los márgenes de este campo de investigación y según la cual las cargas se moverían libremente por el interior de un paisaje formado por bandas de varios átomos de anchura.

Le problème est que la pratique de recourir systématiquement à un terme scientifique peut déboucher, dans certains cas, sur des glissements de sens importants par rapport au texte original, comme le montre l'exemple suivant:

\begin{tabular}{|l|l}
\hline l'avènement des ordinateurs & la aparición de los ordenadores
\end{tabular}

Mais ce n'est qu'avec le développement des réseaux sismologiques et l'avènement des ordinateurs, dans les années 1960, que la preuve définitive de la solidité de la graine sera apportée ${ }^{4}$.

Pero sólo en los años sesenta, con el desarrollo de las redes sismológicas y la aparición de los ordenadores, se consiguió una prueba definitiva de la solidez del núcleo interno.

Dans le texte de géophysique dont est extrait ce passage, "avènement», mot de la langue courante doté d'un grand pouvoir évocateur, peut sembler surprenant. Le dictionnaire Le petit Robert nous en donne la définition suivante:

avènement: 1. (relig. chrét.) arrivée, venue.

2. accession au trône, élévation au pouvoir souverain.

3. (fig.) début du règne de quelque chose.

Dans l'exemple ci-dessus, «avènement », au sens figuré de la troisième acception, reflète très bien le début de cette nouvelle ère de l'informatique dans laquelle nous vivons. Mais en espagnol, il n'y a pas vraiment de mot équivalent pouvant recueillir toutes les connotations du mot français. Dans ce cas, le traducteur, considérant que l'informatique est un phénomène de société, a traduit par "aparición», car le groupe lexical «aparición de un fenómeno» fait partie de ces expressions qui sont si courantes dans le «jargon» scientifique qu'elles fonctionnent presque comme des collocations.

Or, dans ce cas, le problème est que le texte français fait référence aux années 1960, c'est-à-dire au moment où les ordinateurs ont commencé à être utilisés de façon systématique pour traiter de grandes quantités de données scientifiques et non pas au moment où ils ont été inventés, qu’on situe généralement au tout début des années 1950. Aussi, serait-il préférable de traduire par une expression comme «el uso de los ordenadores » qui, même si elle ne reflète pas les connotations de l'original, présente au moins l'avantage de ne pas introduire dans le texte une erreur de décalage historique. 


\subsection{L'agencement des segments introductifs}

Comme nous l'avons déjà signalé, les introductions des articles publiés dans des revues de semi-vulgarisation scientifique ont pour objectif de renforcer le rôle d'accroche des titres, sous-titres et autres éléments paratextuels. Il s'agit d'attirer le lecteur et de l'inciter à lire. Pour ce faire, l'auteur devra recourir à toute une gamme de mécanismes rhétoriques, aussi bien au niveau du contenu qu'au niveau de la forme et plus particulièrement de l'agencement des différents segments.

En ce qui concerne l'agencement des segments, on observe que chaque communauté linguistique semble avoir des préférences pour des formes de mise en texte déterminées et que ces préférences sont souvent différentes d'une langue à une autre. Dans les textes français analysés, on relève une utilisation fréquente de mécanismes comme:

- les questions rhétoriques - parfois même plusieurs questions enchaînées - qui contribuent à la polyphonie caractéristique des introductions:

Quelles sont réellement ces possibilités thérapeutiques? Quelles sont les techniques utilisées? Les cellules souches doivent-elles être assimilées à des embryons, et quelles en seraient les implications? Quelle différence existe-t-il avec le «clonage humain ${ }^{5}$ ?

- les énumérations de segments courts sans verbe:

Nuées de criquets ravageurs, fleuves rougis de sang, chevaux à tête de lion dont les naseaux soufflent le feu et le souffre... la Bible recèle une terrifiante anthologie de calamités, présentées comme des châtiments infligés par un Dieu avide de vengeance ${ }^{6}$.

- les segments antéposés:

Élaborée voici plus de cinquante ans, la théorie synthétique de l'évolution a résisté à la découverte du code génétique, mais porte la marque de son temps ${ }^{7}$.

Ces segments courts, marqués par des signes de ponctuation, donnent à l'introduction un rythme saccadé qui rappelle la langue orale. L'auteur utilise ces mécanismes pour simuler une sorte de dialogue avec le lecteur qu'il tente d'impliquer dans la lecture.

Dans les traductions, ces éléments sont la plupart du temps traduits de façon littérale, ce qui semble un peu forcé. En effet, l'analyse de textes originaux espagnols de ce type montre que les séries de trois et même quatre questions rhétoriques ou l'accumulation de segments courts sans verbe sont très peu fréquentes dans cette langue.

Mais lorsque ces mécanismes sont combinés, la traduction littérale devient impossible et le traducteur doit réorganiser les éléments pour construire un texte d'arrivée plus naturel. Or, on observe que ces réorganisations d'éléments sont souvent accompagnées de suppressions ou de déplacements de certains segments qui ne sont pas sans conséquences:

Relevés au hasard des couvertures de journaux ou des cours de la Bourse, les neuf premiers chiffres de 1 à 9 apparaissent-ils avec des fréquences différentes? À cette question, la plupart des gens répondraient sans hésiter par la négative ${ }^{8}$.

Las nueve primeras cifras, del 1 al 9, ¿aparecen con frecuencias diferentes en los titulares de los periódicos o en las cotizaciones de la bolsa? La mayoría de las personas responderían sin vacilar que no. 
Dans cet exemple, le segment «relevés au hasard» apporte une précision essentielle car l'auteur tente de démontrer que certaines régularités mathématiques surgissent à partir d'éléments divers et aléatoires. Sa position en tête de phrase est donc un mécanisme de mise en relief qui permet d'insister sur cette particularité mathématique. Le traducteur, qui n’a pas reconnu la fonction de ce mécanisme, a supprimé ce segment, appliquant à l'introduction la même stratégie qu'au reste du texte. Mais s'il est vrai que, dans le corps d'un texte, les constructions participiales antéposées ont parfois un simple rôle de reprise, lorsqu'elles sont situées dans l'introduction, elles permettent de présenter de façon synthétique et ordonnée des notions essentielles au niveau thématique. Aussi, si certaines techniques de traduction, comme le fait de placer les expressions de temps en tête de phrase sont généralement de bonnes solutions, l'exemple ci-dessous montre que leur application devra être évaluée au cas par cas.

Grâce au rayonnement laser, on sait aujourd'hui refroidir les atomes jusqu'à réaliser des échantillons denses maintenus à des températures de l'ordre du micro kelvin (un millionième de degré au-dessus du zéro absolu $)^{9}$.

Hoy en día, gracias a la radiación láser, sabemos enfriar átomos hasta realizar unas muestras densas mantenidas a temperaturas del orden del microkelvin (una millonésima de grado por encima del cero absoluto).

Ce passage est l'introduction d'un article sur une utilisation spécifique du laser qui permet d'ouvrir de nouvelles voies dans certains domaines de recherche. Dans le texte français, l'expression «aujourd'hui» est une information secondaire, l'information importante étant précisément les possibilités qu'offre l'utilisation du laser. Dans la traduction espagnole, le fait de placer l'expression de temps «hoy en día» en position initiale, et de reléguer "gracias a la radiación láser» à un second plan, presque en incise, déplace l'effet de focus et donne une vision différente alors qu'on pouvait parfaitement conserver l'ordre des segments en espagnol.

\subsection{Les marques de polyphonie}

Les introductions se caractérisent sans aucun doute par la présence de multiples voix qui s'expriment dans un espace textuel relativement réduit. Cette structure polyphonique est en fait une manifestation de la pluralité de fonctions des introductions. L'une des fonctions principales est, comme nous l'avons signalé, d'attirer l'attention du lecteur en faisant intervenir des «voix» qui appartiennent à un univers de croyances général, à travers des allusions à la philosophie:

Jusqu'à la fin du XIX ${ }^{\mathrm{e}}$ siècle, la mort est restée un sujet de méditation réservé aux philosophes et aux théologiens ${ }^{10}$.

à la religion,

Nuées de criquets ravageurs, fleuves rougis de sang, chevaux à tête de lion dont les naseaux soufflent le feu et le souffre... La Bible recèle une terrifiante anthologie de calamités, présentées comme des châtiments infligés par un Dieu avide de vengeance. Or, dans notre vie de tous les jours, les épidémies ${ }^{11} \ldots$

à la littérature,

N'en déplaise à Jules Verne, la Terre possède en son centre une énorme masse de fer liquide $^{12} \ldots$ 
à l'exotisme,

Lors de rites magiques initiatiques, les Indiens brésiliens Mayoruna appliquent sur leurs plaies les sécrétions de la peau d'une rainette arboricole [...] Symboles mythologiques, littéraires, artistiques et folkloriques, grenouilles et crapauds ont toujours eu la part belle dans les médecines traditionnelles: celles des sorciers et des chamanes de la préhistoire, des prêtres-médecins assyriens et des sociétés européennes de l'époque médiévale. Ainsi les Chinois puis les Romains utilisaient-ils des décoctions ${ }^{13} \ldots$

Mais l'auteur met aussi en scène des énonciateurs qui appartiennent à l'univers de discours spécialisé - souvent comme contrepoint à l'univers de croyances général -, des voix qui expliquent ces phénomènes étranges que le lecteur veut découvrir. Ce sont les spécialistes qui parlent, mais tous ne proposent pas nécessairement la même explication et le débat scientifique s'instaure. Un débat auquel l'auteur de l'article participe. Toutefois, dans l'introduction, on entendra surtout sa voix comme modérateur, comme quelqu'un qui présente les différents points de vue d'une façon relativement impartiale, alors que dans le reste du texte il se prononcera certainement en faveur d'une hypothèse déterminée.

Ce rôle de modérateur ainsi que les différents mécanismes rhétoriques que nous avons relevés dans les introductions réapparaîtront dans les conclusions, établissant entre ces deux espaces un rapport de symétrie.

\section{Analyse des conclusions}

La fonction de la conclusion, comme celle de l'introduction, peut se résumer en trois points :

- synthétiser ce qui a été présenté en insistant sur certains aspects essentiels;

- transmettre au lecteur, dans ce dernier contact, des points de vue, des jugements de valeur, voire des états psychologiques plus élaborés, comme des émotions ou des sentiments;

- convaincre le lecteur du bien-fondé de ce qui a été exposé et l'inviter à suivre le développement des recherches sur le sujet traité.

Ces caractéristiques permettent donc d'affirmer que conclure est une action textuelle spécifique qui laisse son empreinte sur tous les éléments qui la composent. C'est aussi un acte subordonné puisqu'il dépend entièrement de ce qui précède, ce qui le soumet à certaines limitations et à une certaine rigidité du point de vue formel.

La conclusion est un bloc nécessaire et, contrairement à l'introduction, assez bien délimité. Nécessaire parce qu'un texte sans conclusion laisserait le lecteur perplexe et l'absence d'une évaluation finale, qui contribue à hiérarchiser les connaissances exposées, lui donnerait la sensation de n'avoir pas bien capté le contenu global. Et bien délimité car le début de la conclusion est le plus souvent marqué par un connecteur indiquant cet acte de clôture.

Ainsi, si du point de vue de la progression textuelle, les introductions et les conclusions ont en principe une fonction diamétralement opposée, elles gardent en réalité une relation de symétrie. Cette symétrie se manifeste non seulement au niveau thématique et référentiel, mais aussi au niveau rhétorique et énonciatif, et permet d'identifier des marques linguistiques que l'on pourrait considérer prototypiques de ces deux blocs textuels et que nous avons classées en trois grandes catégories: 
1. Les signes qui contribuent à la construction d'un réseau de cohérence spécifique: éléments lexicaux, mécanismes cataphoriques et jeux métaphoriques qui établissent un lien entre le langage spécialisé et le langage général.

2. Les mécanismes d'agencement des séquences, caractérisés assez souvent par la présence de connecteurs.

3. Les marques de polyphonie, spécifiques des conclusions.

\subsection{Le lexique comme ensemble de signes qui contribuent à la construction d'un réseau de cohérence spécifique}

Nous avons signalé, au début de cet article, la tendance à utiliser aussi dans les espaces conclusifs des éléments linguistiques qui transmettent des connotations émotives et intentionnelles plus propres au langage courant qu'à un langage scientifique rigoureux.

Cette tendance, qui avait été illustrée par la traduction de "énigme» par problema, est confirmée par d'autres traductions relevées dans le corpus. Le traducteur semble ainsi enclin à utiliser des éléments appartenant au monde objectif et rigide de la science, dont la conclusion voulait s'éloigner, comme on peut l'observer aussi dans cet autre exemple:

Alors que le texte français introduit volontairement une référence familière au voisinage - avoisinantes - dans l'intention de personnaliser les bactéries étudiées, le traducteur, en utilisant une expression nettement scientifique: las bacterias que comparten el mismo medio, efface ce genre de connotations pour reprendre le langage spécialisé, rigide et froid, que l'auteur voulait éviter.

L'acquisition, par les bactéries avoisinantes, de mécanismes leur permettant de résister à ces substances a amené l'écosystème tellurique à un état d'équilibre ${ }^{14}$.

La adquisición por las bacterias que comparten el mismo medio de mecanismos que les permiten resistir a estas sustancias llevó al ecosistema telúrico a un estado de equilibrio.

\subsection{L'agencement des segments conclusifs}

\subsubsection{La présence de connecteurs}

La stratégie fréquente de marquer le début de la conclusion par un connecteur est une façon explicite d'établir un point d'union entre ce bloc textuel et l'espace central du texte.

Les connecteurs, qui n'ont pas de valeur informative, sont des outils destinés à introduire des instructions argumentatives. Quand ils marquent le début d'une conclusion, leur but fondamental devrait être de récapituler et de reformuler; mais ils peuvent aussi remplir d'autres fonctions.

L'étude du corpus nous a permis d'identifier des solutions de traduction que nous avons classées en deux grands groupes:

i) Le choix par le traducteur d'une marque connective aussi ou même plus nettement conclusive que celle de l'original; ce qui explicite la valeur du segment. 
ii) L'élimination dans la traduction de connecteurs conclusifs présents dans l'original; ce qui débouche sur une «désarticulation» du texte traduit, qui perd sans raison une marque importante, celle du début de la clôture textuelle.

En voici quelques exemples:

i) Explicitation de la valeur conclusive:

Les données analysées dans le corpus vont clairement dans ce sens: les connecteurs à valeur coorientée, situés au début de la conclusion, s’imprègnent de cette valeur récapitulative et conclusive et, parmi les nombreuses possibilités d'équivalence de la langue d'arrivée, le traducteur tend à retenir celles qui ont une valeur très nettement consécutive, récapitulative ou conclusive. C'est le cas de:

\begin{tabular}{|l|l}
\hline enfin & por último \\
\hline
\end{tabular}

Un connecteur aussi polysémique que «enfin» peut être traduit en espagnol par des formules diverses (por fin, en fin, es decir...). Le traducteur du passage suivant a préféré la formule qui suggérait le plus nettement la fonction de clôture: por último.

L'écologie, enfin, est un domaine qui a beaucoup évolué au cours des dernières décennies ${ }^{15}$.

La ecología, por último, es un ámbito que se ha transformado considerablemente.

C'est aussi le cas de:

\begin{tabular}{|l|l|}
\hline donc & $\begin{array}{l}\text { por tanto } \\
\text { por consiguiente }\end{array}$ \\
\hline
\end{tabular}

Comme dans l'exemple précédent, parmi les nombreuses possibilités de traduction du connecteur consécutif «donc» (pues, así pues...), le traducteur a opté pour les expressions les plus nettement consécutives ou récapitulatives comme por consiguiente ou por tanto, qu'il a préférées à pues.

L'acquisition des connaissances au $21^{\mathrm{e}}$ siècle résultera probablement d'un compromis entre le message informatisé le plus abstrait et les techniques les plus spécialisées et les plus performantes: il faudra donc pouvoir graviter entre ces deux pôles ${ }^{16}$.

La adquisición de conocimientos en el siglo XXI será probablemente resultado de una transacción entre el mensaje informatizado más abstracto y las técnicas más especializadas y eficaces: se deberá, por tanto, estar en condiciones de fluctuar entre ambos.

ii) Élimination dans la traduction des connecteurs conclusifs présents dans l'original Cette option de traduction a comme conséquence une destructuration du texte traduit, qui perd l'effet de fermeture présent dans le texte de départ. C'est le cas de l'exemple ci-dessous qui, d'ailleurs, illustre parfaitement le ton métaphorique caractéristique d'un grand nombre de conclusions:

En définitive, la Bible n'était pas loin d'avoir tort: c'est nous-mêmes qui attirons sur nous les calamités - non pas par nos péchés, mais en refusant de prendre garde à nos inquiétudes, de nous fier à notre jugement. ${ }^{17}$ 
La Biblia no estaba lejos de equivocarse: somos nosotros mismos quienes atraemos las calamidades - no por nuestros pecados sino por nuestra negativa a tener en cuenta nuestras inquietudes y confiar en nuestro juicio.

\subsubsection{Autres formules conclusives}

Parmi les mécanismes conclusifs, on relève un nombre important d'éléments anaphoriques, qu'ils soient de type pronominal ou lexical.

Des formules comme «tel», «voilà», et d'autres éléments déictiques peuvent assumer une fonction récapitulative tout à fait adaptée à des stratégies conclusives. C'est le cas du paragraphe suivant qui montre aussi le ton philosophique et moralisant de certaines conclusions:

La plupart des programmes de recherche écologique sont trop courts et limités pour traiter des dimensions aussi importantes. Voilà peut-être la plus grande leçon pour la jeune science qu'est l'écologie ${ }^{18}$.

Ce type de construction exige une adaptation aux formules rhétoriques de l'espagnol que le traducteur ne prend pas toujours en compte. Dans la traduction de ce passage, la construction espagnole est plutôt maladroite et, surtout, trop hésitante pour un effet de clôture:

La mayoría de los programas de investigación ecológica son demasiado cortos y limitados para abordar dimensiones tan importantes. Esta es, quizá, la mejor lección para una ciencia tan joven como es la ecología.

Il serait préférable de recourir à une formule qui marque plus nettement la clôture, comme:

Y ésta es tal vez la mejor lección que la joven ciencia de la ecología puede aprender.

Cette valeur structuratrice et récapitulative est aussi présente dans les anaphores lexicales. En effet, l'anaphore permet de synthétiser en un seul mot un ensemble important d'éléments que l'on qualifie et évalue:

Ces défaillances nous enseignent l'amère leçon que le délicat équilibre entre humains et agents infectieux peut se rompre dans les deux sens ${ }^{19}$.

Dans la traduction espagnole, l'élément anaphorique a été supprimé. Cette perte de lien entre la partie centrale et la clôture donne comme résultat un texte moins structuré et moins clair pour le lecteur:

La amarga lección es que el delicado equilibrio entre los humanos y los agentes infecciosos puede romperse en los dos sentidos.

\subsection{Les marques de polyphonie}

Les conclusions sont aussi des espaces fondamentalement polyphoniques. Comme dans le cas des introductions, l'énonciateur qui prend en charge la conclusion aime introduire des réflexions et des points de vue personnels. Par ailleurs, les conclusions présentent assez souvent une vision optimiste en soulignant les nouvelles voies et les espoirs que le texte laisse entrevoir.

Des expressions comme les suivantes sont donc assez fréquentes: 
La rencontre des lasers et des atomes froids ouvre une voie inexplorée de la chimie ${ }^{20}$.

Il demeure que la compréhension détaillée des liens entre ces deux niveaux majeurs de l'organisation hiérarchique du vivant que sont le programme génétique et la morphologie des individus reste à conquérir ${ }^{21}$.

Parfois, les options des traducteurs ne réussissent pas à rendre des formules nettement conclusives, comme dans le cas de la traduction de l'exemple ci-dessus:

Queda todavía lograr la comprensión detallada de las relaciones entre estos dos importantes niveles de la organización jerárquica de los seres vivos que son el programa genérico y la morfología de los individuos.

qui n'arrive pas à exprimer d'une façon nette cette idée «d'une conquête qui reste à faire».

La conclusion demanderait une rédaction plus claire du type:

Por consiguiente, la comprensión detallada de las relaciones entre estos dos importantes niveles de la organización jerárquica de los seres vivos que son el programa genérico y la morfología de los individuos sigue siendo una conquista pendiente.

D’autres jeux polyphoniques, comme les citations ou les questions enchaînées, sont aussi fréquents comme le montre cet exemple:

Que devra-t-on en déduire? La grande chasse touche-t-elle à sa fin? Commence-t-on à détecter des objets vraiment primordiaux? A-t-on enfin affaire à la génération d'étoiles à l'origine des premières poussières? Ou bien aperçoit-on cette raie à travers des «trous » transperçant les nuages de galaxies déjà poussiéreuses ${ }^{22}$ ?

que le traducteur reprend d'une façon littérale:

¿Qué hay que deducir? ¿Llega a su final la gran cacería? ¿Se empiezan a detectar objetos realmente primordiales? ¿Se está finalmente ante la generación de estrellas que originó el primer polvo ? ¿O bien se percibe esta raya a través de «agujeros» que atraviesan las nubes de galaxias ya polvorientas?

Tous ces phénomènes polyphoniques peuvent être combinés de différentes manières. Ainsi, dans l'exemple ci-dessous, l'allusion au Siècle des Lumières - que les guillemets transforment en un élément de polyphonie montrée (Authier-Revuz: 1982) - donne une coloration socioculturelle particulière à la clôture du texte.

Nous pensons que ces bénéfices et d'autres encore issus du clonage et du transfert des noyaux l'emportent de loin sur les dommages éventuels qu'ils pourraient impliquer. Ils ne pourront cependant être obtenus qu'au travers d'une détermination expérimentale. En dernier ressort, ce sera à la société de décider quelle voie emprunter. Espérons que la décision sera «éclairée» et ne se fondera ni sur des peurs irrationnelles ni sur l'ignorance ou les préjugés ${ }^{23}$.

Mais l'allusion culturelle n'a pas été suffisamment prise en compte par le traducteur qui a fait disparaître cette marque du texte et, partant, toutes les connotations et l'intentionnalité dont elle était chargée.

Creemos que estos beneficios y otros emanados de la clonación y de la transferencia de núcleos superan con mucho los daños eventuales que pudieran derivarse. Sin embargo, estos beneficios sólo se podrán obtener a través de una determinación experimental. En último término, corresponderá a la sociedad decidir qué camino quiere emprender. 
Esperemos que la decisión sea clara y que no se base ni en temores irracionales ni en la ignorancia o los prejuicios.

Le but de notre réflexion était de mettre en évidence la spécificité des espaces introductifs et conclusifs, deux blocs textuels dans lesquels l'auteur utilise et combine les mots de façon à mettre en œuvre une fonction résolument argumentative visant à convaincre le lecteur du bien-fondé de ce qui a été exposé. Nous avons voulu souligner aussi que cette fonction peut être contrôlée et analysée car elle laisse des traces facilement repérables dans le discours, comme le choix de certains éléments lexicaux, l'emploi de certains connecteurs, la présence des formules polyphoniques. Tous ces éléments permettent de définir des stratégies de traduction spécifiques lorsqu'on doit reconstruire le sens du texte dans une autre langue.

Les données des traductions analysées montrent un traitement du traducteur qui n'a pas été capable d'évaluer la fonction de ces éléments et de rendre toute l'intentionnalité du texte de départ, les solutions présentées dans les textes traduits ayant, au contraire, neutralisé la valeur de ces outils argumentatifs.

Nous pensons que ce travail pourrait avoir des applications non seulement dans la pédagogie de la traduction, domaine dans lequel nous nous situons, mais aussi dans le cadre plus large des processus de lecture et écriture textuelles. Elles pourraient aussi être extrapolées à d'autres typologies textuelles.

Du point de vue de la méthodologie de la traduction, il est évident que, dans l'activité d'interprétation et de reconstitution d'un texte dans une autre langue, les stratégies discursives qui ont donné lieu au texte de départ jouent un rôle aussi important que le message à transmettre. L'enseignement de cette matière devrait donc tenir compte de toutes les nuances présentes dans ces stratégies, car seule une évaluation correcte de l'ensemble des enjeux textuels permettra de construire un acte communicatif qui produise sur le lecteur un effet équivalent.

\section{NOTES}

1. A. Souriau «Le noyau de la terre, tourne-t-il vraiment?», La Recherche, 315, décembre 1998. « ¿Gira realmente el núcleo de la tierra?», Mundo científico, 198, febrero 1999.

2. A. Souriau «Le noyau de la terre, tourne-t-il vraiment?», La Recherche, 315, décembre 1998. «¿Gira realmente el núcleo de la tierra?», Mundo científico, 198, febrero 1999.

3. R. Service, "La supraconductivité en bandes», La Recherche, 320, mai 1999. "Superconductividad en bandas», Mundo Científico, 203, julio/agosto 1999.

4. A. Souriau «Le noyau de la terre, tourne-t-il vraiment?», La Recherche, 315, décembre 1998. «¿Gira realmente el núcleo de la tierra?», Mundo científico, 198, febrero 1999.

5. D. Solter et J. Gearhart, "Des cellules bonnes à tout faire?», La Recherche, 320, mai 1999. «¿Células aptas para todo?» Mundo Científico, 203, julio/agosto 1999.

6. G. Armelagos, «Des réseaux planétaires pour les microbes», La Recherche, 314, novembre 1998 «Redes planetarias para los microbios», Mundo Científico, 197, enero 1999.

7. J. Chaline, D. Marchand, «Quand l'évolution change le temps des êtres», La Recherche, 316, janvier 1999.

«Cuando la evolución cambia el tiempo de los seres», Mundo Científico, 199, marzo 1999.

8. T. Hill, «Le premier chiffre significatif fait sa loi», La Recherche, 316, janvier 1999. «La primera cifra significativa dicta su ley», Mundo Científico, 199, marzo 1999

9. P. Pillet, F. Masnou-Seeuws, «Jeu de photons pour chimie froide» La Recherche, 318, mars 1999. "Juego de fotones para la química fría», Mundo Científico, 201, mayo 1999.

10. H. Denis, «L'immortalité des cellules sexuelles», La Recherche, 322, juillet-août 1999.

11. G. Armelagos, «Des réseaux planétaires pour les microbes», La Recherche, 314, novembre 1998. «Redes planetarias para los microbios», Mundo Científico, 197, enero 1999. 
12. R. Jeanloz, B. Romanowicz, «Le noyau de la Terre: et pourtant il tourne... », La Recherche, 308, avril 1998.

13. M. Amiche, A. Dufour et P. Nicolas, «Les trésors de la peau des grenouilles», La Recherche, 291, octobre 1996

14. R. Trieu-Cuot, C. Poyart «Visite guidée au cœur de l'arsenal bactérien», La Recherche, 314, novembre 1998.

«Visita guiada al centro del arsenal bacteriano», Mundo Científico, 197, enero 1999.

15. A. Deleon, «Un quart de siècle après », Le Courrier de l'Unesco, janvier 1997.

«Hace un cuarto de siglo», El Correo de la Unesco, enero 1997.

16. F. Gros, «Décloisonner la science», Le Courrier de l’Unesco, septembre 1997. «Descompartimentar la Ciencia», El Correo de la Unesco, septiembre 1997.

17. G. Armelagos, «Des réseaux planétaires pour les microbes », La Recherche, 314, novembre 1998. «Redes planetarias para los microbios», Mundo Científico, 197, enero 1999.

18. W.K. Stevens, "Loutres perdues en mer», La Recherche, 318, mars 1999. «Nutrias perdidas en el mar», Mundo Científico, 201, mayo 1999.

19. G. Armelagos, «Des réseaux planétaires pour les microbes », La Recherche, 314, novembre 1998. «Redes planetarias para los microbios», Mundo Científico, 197, enero 1999.

20. P. Pillet, F. Masnou-Seeuws, «Jeu de photons pour chimie froide» La Recherche, 318, mars 1999. «Juego de fotones para la química fría», Mundo Científico, 201, mayo 1999.

21. J. Chaline, D. Marchand, «Quand l'évolution change le temps des êtres», La Recherche, 316, janvier 1999.

«Cuando la evolución cambia el tiempo de los seres», Mundo Científico, 199, marzo 1999.

22. B.Guiderdoni, F.R.Bouchet, "Les chasseurs de galaxies primordiales", La Recherche, 318, mars 1999.

«Los cazadores de galaxias primordiales», Mundo Científico, 201, mayo 1999.

23. D.Solter et J. Gearhart, «Des cellules bonnes à tout faire?», La Recherche, 320, mai 1999. «¿Células aptas para todo?», Mundo Científico, 203, julio/agosto 1999.

\section{RÉFÉRENCES}

ADAm, J.M. (1992): Les textes: types et prototypes, Paris, Nathan.

AdAm, J.M. (1997): L'argumentation publicitaire. Rhétorique de l'éloge et de la persuasion, Paris, Nathan.

Anscombre, J.C. (1989): "Théorie de l'argumentation, topoï et structuration discursive», Revue québécoise de linguistique.

Anscombre, J.C. (1995): «La nature des topoï», Théorie des topoï, Paris, Editions Kimé.

Anscombre, J.C. et O. Ducrot (1983): L'argumentation dans la langue, Bruxelles, Mardaga.

Ducrot, O. (1982): «Note sur l'argumentation et l'art d'argumenter», Cahiers de Linguistique Française 4, Université de Genève.

Ducrot, O. (1984): Les mots du discours, Paris, Minuit.

Ducrot, O. (1984): Le dire et le dit, Paris, Minuit.

Ducrot, O. (2001) : "Critères argumentatifs et analyse lexicale», Langages 142, p. 22-40.

JАсові, D.(1987): Textes et images de la vulgarisation scientifique, Berne, Peter Lang.

Maingueneau (1987): Nouvelles tendances en analyse du discours, Paris, Hachette.

Perelman, C. et L. Olbrechts-Tyteca (1988): Traité de l'argumentation, Bruxelles, Éditions de l'Université de Bruxelles.

Portolés, J. (1998): Marcadores del discurso, Barcelona, Ariel.

RACСAн, P.Y. (1990): "Signification, sens et connaissance: une approche topique», Cahiers de Linguistique Française 11, Université de Genève.

RACCAH, P.Y. (1995) : «Argumentation and natural language: Presentation and discussion of four foundational hypotheses », Journal of Pragmatics 24-1/2.

RaсcAн, P.Y. (1998): "Lexical and dynamic topoi in semantic description: A theoretical and practical differentiation between words and terms", In: Language, Text and Knowledge (eds Lundquist et al.), Mouton. 
RACCAH, P.Y. (1998): «Argumentation and Knowledge: From Words to Terms», In: Incommensurability and Translation (eds.: Rossini, Sandri et Scazzieri), Cheltenham (UK), Elgar.

Rey-Debove, J. et A. Rey (1993): Le Nouveau Petit Robert 1. Dictionnaire alphabétique et analogique de la langue française, Paris, Dictionnaires Le Robert.

ReY, J. (1998 ) : «La argumentación en la divulgación científica», Escritos 17/18.

Rey, J. (2000): «La traduction des textes scientifiques: structure textuelle et processus cognitifs», Target 12-1, p. 63-82.

Rubbatel (1982): «De la syntaxe des connecteurs pragmatiques», Cahiers de Linguistique Française 11, Université de Genève.

Tricás, M. (1991): «Enfin, j'ai compris!» (les valeurs pragmatiques du connecteur et sa traduction), Méthodologie, formation, pragmatique et analyse textuelle, Institut de Ciències de l'Educació, Universitat Autònoma de Barcelona.

TricÁs, M. (1995): Manual de Traducción, Barcelona, Gedisa.

Tricás, M. (2001): "La traduction à l'espagnol de quelques connecteurs de juxtaposition», Langages 143, Paris, Larousse.

Tricás, M. (2002): «Del Universo de creencias al texto. Reflexiones sobre estrategias interpretativas en la construcción del sentido", Cartografías de la Traducción. Del post-estructuralismo al multiculturalismo, Román Álvarez (ed.) Biblioteca traducción, Ediciones Almar, p. 279-297. 